\title{
Thanking Cancer Cell International's 2013 reviewers
}

Denys Wheatley

\section{Contributing reviewers}

The Editor-in-Chief would like to thank all the reviewers who contributed to the journal in 2013.

Adrian Britschgi

Switzerland

Ai-ling Tian

China

Ajay Bommareddy

United States of America

Alberto Ferlin

Italy

Alessia Ongaro

Italy

Alexander Dobrovic

Australia

Alfredo De Paula

Brazil

Alok Mishra

United States of America

Amanda Ward

United States of America

Amanda Dixon-McIver

New Zealand

Ammad Farooqi

Pakistan

Anália Carmo

Portugal

Andrea Rasola

Italy
Andreas Villunger

Austria

Anna Litwiniec

Poland

Anna Friedl

Germany

Anthony Gavalas

Greece

Anthony Gavalas

Germany

Antoinette Hollestelle

Netherlands

Antonella Canini

Italy

Anwar Hossain

United States of America

Aras Emre Canda

Turkey

Ariana Bruzzone

Argentina

Ashraf Abdel-Naim

Saudi Arabia

Axel Hegele

Germany

Baoan Chen

China
Basavaraj Koti

India

Bassel El-Rayes

United States of America

Ben Loos

South Africa

Benedetta Bussolati

Italy

Bilge Sener

Turkey

Bin Shan

United States of America

Bo Cao

United States of America

Bo Guang Fan

Canada

Bonhong Min

Korea, South

Boris Zhivotovsky

Sweden

Boris Negrutskii

Ukraine

Boudewijn J Braakhuis

Netherlands

Bruno Robbs

Brazil 


\begin{tabular}{|c|c|c|}
\hline $\begin{array}{l}\text { Bruno Vincenzi } \\
\text { Italy }\end{array}$ & $\begin{array}{l}\text { Deodutta Roy } \\
\text { United States of America }\end{array}$ & $\begin{array}{l}\text { Farah Khan } \\
\text { India }\end{array}$ \\
\hline Bulent Ozpolat & Dick Mosser & Federica Sotgia \\
\hline United States of America & Canada & United Kingdom \\
\hline Byong Chul Yoo & Dihua Shangguan & Fiona Yull \\
\hline Korea, South & China & United States of America \\
\hline Cameron Bracken & Dominique Modrowski & Francois Martin \\
\hline Australia & France & France \\
\hline Cecilia Hegardt & Dongjun Peng & Frank Greco \\
\hline Sweden & United States of America & United States of America \\
\hline Cedric Rebe & Douglas Demetrick & Fraz Awan \\
\hline France & Canada & Pakistan \\
\hline Chandramu Chetty & Douglas Hurst & Gabriella D'Orazi \\
\hline United States of America & United States of America & Kazakhstan \\
\hline Chin-Cheng Su & Dustin Deming & Gabriella Pagnan \\
\hline Taiwan & United States of America & Italy \\
\hline Christer Thomsen & Elena Takano & Galatea Kallergi \\
\hline Sweden & Australia & Greece \\
\hline Christian Stock & Elena Nadezhdina & Gangadhara Sareddy \\
\hline Germany & Russian Federation & United States of America \\
\hline Christiane Maier & Elif Damla Arisan & Gar-Yang Chau \\
\hline Germany & Turkey & Taiwan \\
\hline Daigo Sumi & Elizabeth Guo & Geert Bultynck \\
\hline Japan & China & Belgium \\
\hline Daisuke Ennishi & Ellen Heitzer & George Calin \\
\hline Canada & Austria & United States of America \\
\hline Daniel Ramon Ciocca & Elsa Bronze-da-Rocha & Georgios Nteliopoulos \\
\hline Argentina & Portugal & United Kingdom \\
\hline Daniela Gallo & Emilio Gonzalez & Gian Luigi Russo \\
\hline Italy & Spain & Italy \\
\hline Daniele Vergara & Erhong Meng & Giulio Metro \\
\hline Italy & United States of America & Italy \\
\hline Darell Bigner & Esra Erdal & Gloria Calaf \\
\hline United States of America & Turkey & United States of America \\
\hline David Buxton & Eumorphia Konstantakou & Grigori Rychkov \\
\hline United States of America & Greece & Australia \\
\hline David Emlet & Ewa Balcerczak & Guixing Qiu \\
\hline United States of America & Poland & China \\
\hline Degang Shi & Fabio Luis Forti & Gulgun Oktay \\
\hline China & Brazil & Turkey \\
\hline Delia Mezzanzanica & Fan Yi & Guochang $\mathrm{Hu}$ \\
\hline Italy & China & United States of America \\
\hline Delong Liu & Fang Yan & Guoyou Zhang \\
\hline United States of America & United States of America & Australia \\
\hline
\end{tabular}




\author{
Haiteng Deng \\ China \\ Hans-Joachim Mueller \\ Germany \\ Hark Kim \\ Korea, South \\ Hecheng Li \\ China \\ Helfrid Hochegger \\ United Kingdom \\ Hong Fan \\ China \\ Hong Chang \\ Canada
}

Hong He

United States of America

Hong Yang

China

Hong He

Australia

Hong Zheng

United States of America

Hongmei Nan

United States of America

Hong-Sheng Zhang

China

Hongwei Zhang

China

Hongwei Li

China

Huifang Hao

Japan

Ido Wolf

Israel

Indu Kohaar

United States of America

Irfan Asangani

United States of America

Isabel Andujar

Spain

Isabelle Dhennin-Duthille

France

Istvan Zupko

Hungary
Istvan Vermes

Netherlands

James A McCaul

United Kingdom

Jamie Jarboe

United States of America

Jean Koff

United States of America

Jekaterina Erenpreisa

Latvia

Jenifer Prosperi

United States of America

Jessy Deshane

United States of America

Jia Xu

United States of America

Jiahua Zhu

United States of America

Jiajun Shi

United States of America

Jing Lin

United States of America

Jiri Vrba

Czech Republic

Joana Carvalho

Portugal

John Pulikkan

Germany

Jong Bin Kim

Korea, South

Jong Kuk Park

Korea, South

Jun Yao

China

Jun Yasuda

Japan

Jun Yang

United States of America

Junaid Abdulghani

United States of America

June Zou

United States of America

Junming Yue

United States of America
Junming Guo

China

Justine Rudner

Germany

Kajsa Paulsson

Sweden

Kaleeckal Harikumar

United States of America

Kameswaran Ravichandran

United States of America

Karine Breckpot

Belgium

Karl Chai

United States of America

Katharine Irvine

Australia

Kazuhiro Kunimasa

Japan

Keizo Takenaga

Japan

Ken Young

United States of America

Ken-ichi Ogawara

Japan

Kevin Sun

New Zealand

Konradin Metze

Brazil

Kotb Abdelmohsen

United States of America

Kwadwo Asamoah Kusi

Ghana

Li Xiaorong

China

Li Cao

United States of America

Liguang Chen

United States of America

Ling Wang

China

Liping Liu

China

Lisa Murphy

Ireland 


\begin{tabular}{|c|c|c|}
\hline Lizong Shen & Mehdi Shafa & Natarajan Aravindan \\
\hline China & Canada & United States of America \\
\hline Ljubica Vucicevic & Meixia Zhang & Natasha Kyprianou \\
\hline Serbia & China & United States of America \\
\hline Long-Cheng Li & Meng Qingbing & Nathalie Johnson \\
\hline United States of America & China & Canada \\
\hline Lorenzo Sempere & Meng-Ru Shen & Navasona Krishnan \\
\hline United States of America & Taiwan & United States of America \\
\hline Luc Dirix & Michael Chan & Nayoung Kim \\
\hline Belgium & Taiwan & Korea, South \\
\hline Lucia Sfondrini & Michael Graner & Neelu Puri \\
\hline Italy & United States of America & United States of America \\
\hline Luis Goya & Michele Caraglia & Nicholas Pavlidis \\
\hline Spain & Italy & Greece \\
\hline Lyuba Varticovski & Mike Fainzilber & Nicola Aceto \\
\hline United States of America & Iran & United States of America \\
\hline Magda hagras Saudi & Ming Yuan & Nives Pecina-Slaus \\
\hline Arabia & United Kingdom & Croatia \\
\hline Makoto Makishima & Minghua Wu & Nizar Mhaidat \\
\hline Japan & China & Jordan \\
\hline Manxiang Li & Mitsuho Onimaru & Ola Hermanson \\
\hline China & Japan & Sweden \\
\hline Marco Cerbón & Mohamed Saleh Mahmoud & Oleh Stasyk \\
\hline Mexico & Canada & Ukraine \\
\hline Maria Teresa Teresa & Mohit Sachdeva & Oliver Treeck \\
\hline Italy & United States of America & Germany \\
\hline Marius Raica & Mu Zhang & Omar Mohafez \\
\hline Romania & United States of America & Japan \\
\hline Martin Liu & Mugdha Khaladkar & Osamu Miura \\
\hline United States of America & United States of America & Japan \\
\hline Martino Introna & Munirathinam Gnanasekar & Pao-Ling Torng \\
\hline Italy & United States of America & Taiwan \\
\hline Maryna de Kock & Muriel Priault & Paolo Aurello \\
\hline South Africa & France & Italy \\
\hline Massimo Derenzini & Muthusamy Kunnimalaiyaan & Paolo Martini \\
\hline Italy & United States of America & United States of America \\
\hline Massimo Aglietta & Nahum Sonenberg & Paolo Gandellini \\
\hline Italy & Canada & Italy \\
\hline Massimo Zollo & Natalia Ceaglio & Parvin Mehdipour \\
\hline Italy & Argentina & Iran \\
\hline Mayuko Furuta & Natalija Eigeliene & Patricio Aller \\
\hline Japan & Finland & Spain \\
\hline
\end{tabular}


Paul Agutter

United Kingdom

Paul Bollyky

United States of America

Pavel Soucek

Czech Republic

Pedro P. Lopez-Casas

Spain

Peiman Liu

United States of America

Peiyu Pu

China

Penka Nikolova

United Kingdom

Periasamy Selvaraj

United States of America

Peter Schraml

Switzerland

Peter Olbert

Germany

Phyllis Wachsberger

United States of America

Pier Giorgio Petronini

Italy

Ping Zhu

China

Ping-an He

China

Po-Wu Gean

Taiwan

Qian Xu

China

Qing-Hua Meng

China

Qingsong Zhu

United States of America

Rajakishore Mishra

United States of America

Rajendra Sharma

Canada

Rakesh Naidu

Malaysia
Ramon Alemany

Spain

Ravindresh Chhabra

India

Rik Derynck

United States of America

Rishi Chhipa

India

Robert Milewski

Poland

Rohit Malik

United States of America

Roland Lauster

Germany

Romain Debret

France

Roman Eliseev

United States of America

Ruben Plentz

Germany

Sabine Hombach-Klonisch

Canada

Salvatore Pizzo

United States of America

Samer Hakim

Germany

Sander van den Driesche

United Kingdom

Sandra Karlsson

Sweden

Sarah Prior

United Kingdom

Saskia Cillessen

Netherlands

Sen Zhang

China

Shaofang $W u$

United States of America

Sheng-mei Zhu

China

Shigeki Ito

Japan
Shounanyi Yi

Australia

Shuyu Ren

United States of America

Sidney Yu

Hong Kong

Smadar Avigad

Israel

Song YQ

China

Sreenivasulu Chintala

United States of America

Stefan Pusch

Germany

Stephan Reshkin

Italy

Stephanie Vispe

France

Steve Chambers

New Zealand

Steven Van Laere

Belgium

Sudhakar Veeranki

United States of America

Suoqin Tang

China

Suresh Tikoo

Canada

Sydney Mukaratirwa

United Kingdom

Tadashi Kondo

Japan

Tae il Kim

Korea, South

Takatsune Shimizu

Japan

Takeshi Miyamoto

Japan

Takuya Moriya

Japan

Tao Ren

China 
Tao Lin

United States of America

Taodi Liu

China

Tatiana Zybina

Russian Federation

Terry Moody

United States of America

Thomas Scheper

Germany

Tiago Rodrigues

Brazil

Timothy Gershon

United States of America

Toni Ibrahim

Italy

Tonya Walser

United States of America

Tsui-Ling Chang

Taiwan

Tsutomu Shimura

Japan

Ugo Testa

Italy

Ulrich Renner

Germany

Ulrike Stein

Germany

Umberto Galderisi

Italy

Urmila Santanam

United States of America

Urs Lichtenauer

Germany

Valentina Todorova

United States of America

Valery Grdzelishvili

United States of America

Vishal Gupta

India

Vladimir Pushkarev

Ukraine
Vladimir Gogvadze

Sweden

W. Keith Miskimins

United States of America

Wanming Zhao

United States of America

Wei Du

United States of America

Wei Ding

China

Weili Cai

United States of America

Wen Zhuang

China

Wen Ying Wu

China

Wendy Hwang-Verslues

Taiwan

Wenjun Wang

China

Wenling Wang

China

Wenxi Wu

China

Wenyue Sun

United States of America

William Cho

Hong Kong

Wolfram Miekisch

Germany

Wu Dong-Cheng

China

Xiaobin Jia

China

Xiaobing He

United States of America

Xiaobo Li

United States of America

Xiaochun Xu

United States of America

Xiaohua Jiang

Hong Kong
Xiaoping Miao

China

Xiaoqing Niu

China

Xianoyong Zheng

United States of America

Xin-xia Tian

China

Xiuwu Zhang

United States of America

Yajuan Su

China

Yan Huang

United States of America

Yanfeng Qi

United States of America

Yang Yao

China

Yang-Chang Wu

Taiwan

Yangqiu Li

China

Yei-Tsung Chen

Singapore

Yen-Ni Teng

Taiwan

Yi Zhang

China

Yiannis Drosos

United States of America

Ying Jin

China

Yingpeng Liu

United States of America

Ymera Pignochino

Italy

Yoji Nagashima

Japan

Yong Yang

China

Yongwen Chen

China 


\section{Yoshiki Murakami}

Japan

Yoshio Honma

Japan

Yu Liu

United States of America

Yu Jin

United States of America

Yunge Zhao

United States of America

Zahid Siddik

United States of America
Zeyi Zheng

United States of America

Zhaoxiang Bian

Hong Kong

Zhi Wang

China

Zhi-guang Tu

China

\section{Zhi-Hong Wen}

Taiwan

Zhijun Dai

China
Zihua Zeng

United States of America

Zongbing You

United States of America

doi:10.1186/1475-2867-14-11

Cite this article as: Wheatley: Thanking

Cancer Cell International's 2013 reviewers.

Cancer Cell International 2014 14:11. 